\title{
A Case of Angioid Streaks in Congenital Dyserythropoietic Anaemia Type II
}

\author{
Emer Doolan Andrea Ryan \\ Ophthalmology Department, Royal Victoria Eye and Ear Hospital, Dublin, Ireland
}

\section{Keywords}

Angioid streaks · Congenital dyserythropoietic anaemia

\begin{abstract}
The authors describe a case report of retinal angioid streaks (AS) in a patient with congenital dyserythropoietic anaemia (CDA) type II and compare the retinal findings to those of an affected first-degree relative without ocular manifestations of the disease. A 52-year-old man with a confirmed diagnosis of CDA type II has been dependent on treatment with regular transfusions and chelating agents. He presents with bilateral retinal AS. The subject's brother, who also has CDA type II, underwent splenectomy in childhood, and has required no treatment since then. He has no ocular manifestations of the disease. To the authors' knowledge, this is only the second time that the presence of retinal AS has been reported in a case of CDA type II. It has been reported more frequently with CDA types I and III. The milder course of disease in the subject's brother likely accounts for the differing retinal findings. The authors explore the pathophysiology of AS in this disease, and the differential diagnosis of chelating agent toxicity. Diagnostic uncertainty around retinal findings can lead to withholding of essential systemic treatment and inappropriate ophthalmological follow-up. It is recommended that all patients with CDA undergo eye examinations.
\end{abstract}

\section{Karger $\stackrel{2}{\circ}$}




\section{Introduction}

Angioid streaks (AS) represent visible irregular crack-like dehiscences in Bruch's membrane (BM) that are sometimes associated with atrophic degeneration of the overlying retinal pigment epithelium (RPE) [1] and a break in or absence of choriocapillaris underneath. They are almost always bilateral and radiate from the peripapillary area. AS have been reported most commonly as an ocular manifestation of pseudoxanthoma elasticum (PXE) and Paget's disease. AS reportedly occur in $59-87 \%$ of PXE patients and $8-15 \%$ of patients with Paget's disease. Other common systemic associations are Ehlers-Danlos syndrome, Marfan syndrome, haemoglobinopathies including sickle cell disease and thalassaemia major, and hypercalcaemia. The incidence of AS in sickle cell haemoglobinopathies is $0.9-6 \%$ depending on study size [2]. There are many other less common potential systemic associations. In general, the onset of AS commonly lies between the second and fifth decades, depending also on the underlying systemic disease.

AS are largely asymptomatic. Symptoms of metamorphopsia or reduced visual acuity occur if AS involve the fovea or if the potentially devastating complication a of choroidal neovascular membrane (CNVM) occurs. The incidence of CNVM in AS patients varies by study but is reported to be as high as $42-86 \%$ in studies where the majority of subjects had PXE [2]. The risk of developing CNVM increases with age, and also with width and length of the AS [2]. The risk of developing CNVM may be lower in patients with AS secondary to haemoglobinopathy, as only 2 of 21 eyes with AS in a study of homozygous sickle cell disease had findings consistent with CNVM [3]. Where CNVM develops, the untreated prognosis is poor. Intravitreal anti-VEGF is the treatment of choice, with small studies showing successful outcomes with bevacizumab and ranibizumab [2].

AS were first reported as an ocular manifestation of congenital dyserythropoietic anaemia (CDA) in 1997, when they were incidentally discovered in the eyes of 6 members of a family affected with CDA type III [4]. They were later reported in CDA I [5-7] and most recently in CDA II [7]. The term dyserythropoiesis refers to a condition of abnormal erythropoiesis affecting the differentiation and proliferation pathways of red blood cells. CDA is a rare hereditary disease, with prevalence varying among European populations. There are 0.04 cases/ million in Northern Europe and 2.49/million in Mediterranean countries [8].

The 3 classical types of CDAs (types I, II, and III) are defined by bone marrow morphology. They are highly heterogeneous; genetically, and phenotypically. CDAI is inherited in an autosomal recessive variant in either the CDAN1 gene on chromosome 15q.15.2 or the CDIN1 gene on chromosome 15q.4. CDA II is the most common type and is characterized by autosomal recessive variants in the SEC23B gene on chromosome 20p11.23. CDA III is the least common of the classical types. It is inherited predominantly in an autosomal dominant manner, although sporadic cases have been reported. The gene involved in the KIF23 gene on chromosome 15q21 [7].

The clinical picture of CDA includes variable degrees of anaemia, iron overload, jaundice, splenomegaly, hepatomegaly, and dysmorphisms predominantly affecting the digits. Most have mild-moderate anaemia only. Some severely affected patients remain transfusion dependent or require splenectomy [7].

\section{Case Report}

We describe the case of a 52-year-old man referred for the opinion of a medical retina specialist due to possible retinal desferrioxamine (DFO)/deferasirox toxicity. He was diagnosed with CDA type II in childhood and was under regular review by haematology. His

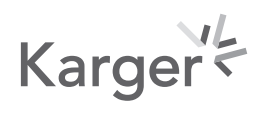



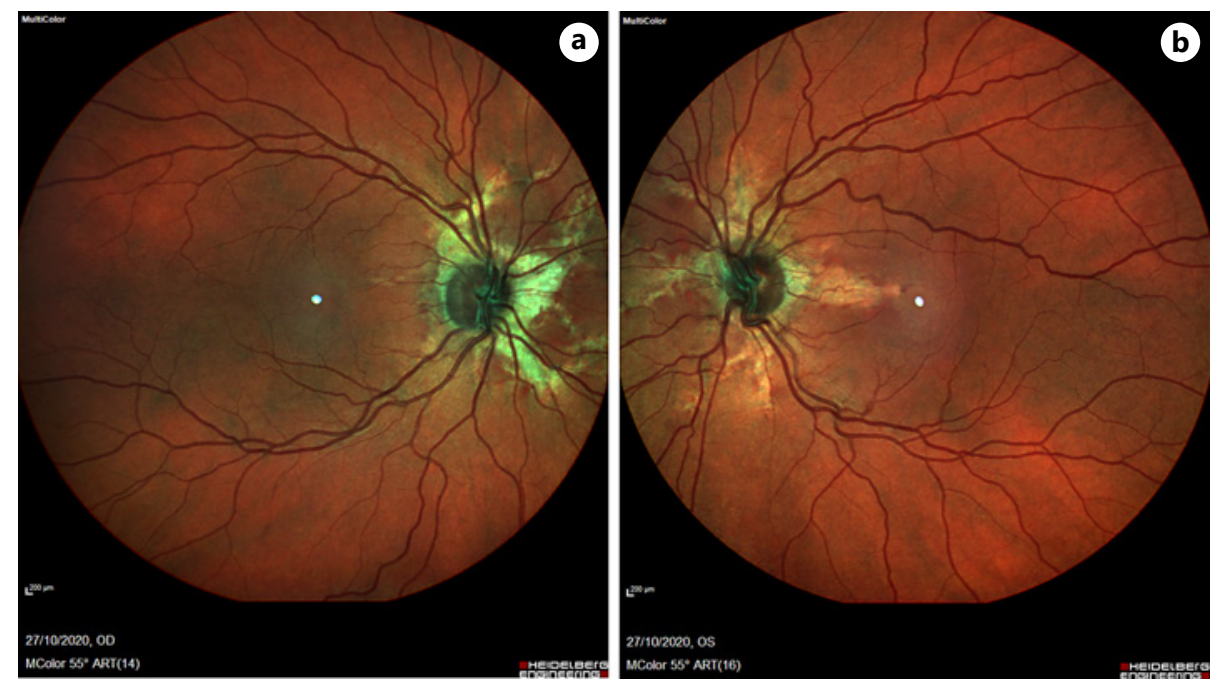

Fig. 1. a Confocal scanning laser ophthalmoscopy (CSLO) composite colour image of the subject's right eye showing angioid streaks. b CSLO composite colour image of the subject's left eye showing AS.

course had been complicated by hypersplenism, persistent anaemia, iron overload, and an aplastic crisis secondary to Parvovirus B. He had in the past been treated with both of the abovementioned chelating agents in conjunction with regular blood transfusions. He had not required any surgical intervention.

His treatment had recently been withheld due to concerns over retinal toxicity which had been diagnosed and monitored over a 10-year period by an ophthalmologist in another centre. The retinal findings reported by the previous ophthalmologist were "degenerative changes" bilaterally, which on serial fundus photography over 10 years at another centre were extending towards the left fovea. His haematology consultant wished to recommence treatment pending a report on his retinal findings. He subjectively had no visual complaints. Ocular history was significant for myopia only. Visual acuity was $6 / 6$ in both eyes. On examination, he had bilateral AS. There was no associated CNVM. This was established by spectraldomain optical coherence tomography (SD-OCT) and later by OCT angiography. The images obtained by the previous ophthalmologist were unavailable for comparison.

Confocal scanning laser ophthalmoscope (CSLO) composite colour images of both eyes show pale striated lesions radiating from the optic nerves (shown in Fig. 1a, b). CSLO fundus autofluorescence (FAF) images of both eyes show hypoautofluorescent fissures indicative of the attenuated or absent RPE associated with the AS (shown in Fig. 2a, b). SD-OCT of the left eye localized over an AS shows BM undulation and adjacent BM break (shown in Fig. 3).

Electrodiagnostic testing, in the form of a full-field (Ganzfeld) electroretinogram, was carried out. It was reported as having normal rod-isolated and con-isolated responses in both eyes, with no evidence of significant pan-retinal photoreceptor dysfunction. Notably, none of the imaging or electrodiagnostic testing findings associated with DFO toxicity (see Discussion) were identified. After 1 year of follow-up, retinal findings were unchanged with no evidence of CNVM and no change in VA.

The subject had one sibling affected with CDA II, a brother who was treated with DFO in childhood but had required no treatment at all since undergoing a splenectomy aged 8 . He had no retinal manifestations of the disease. CSLO composite colour images of both eyes were unremarkable (shown in Fig. 4a, b). A family pedigree shows our subject's brother (proband) and his brother to be the only affected family members (shown in Fig. 5). 


\section{Case Reports in Ophthalmology}
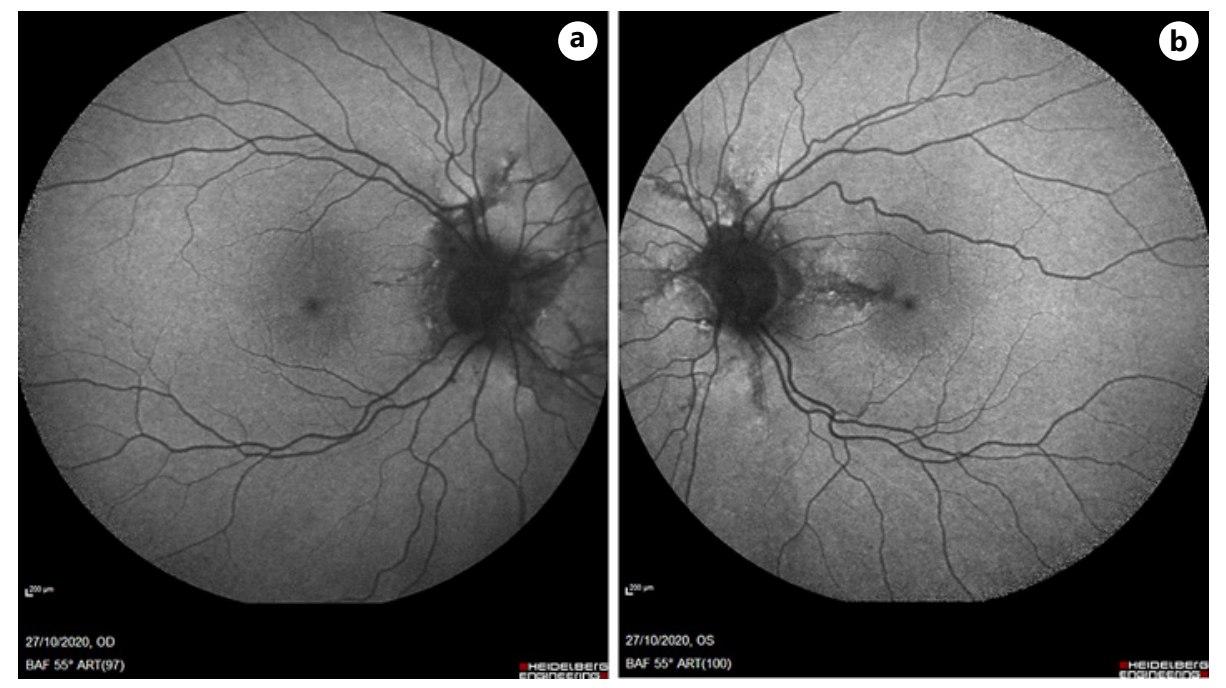

Fig. 2. a Confocal scannling laser ophthalmoscopy (CSLO) autofluorescent images of the subject's right eye showing hypofluorescent areas corresponding to the angioid streaks (AS). b CSLO autofluorescent images of the subject's left eye showing hypofluorescent areas corresponding to the AS.

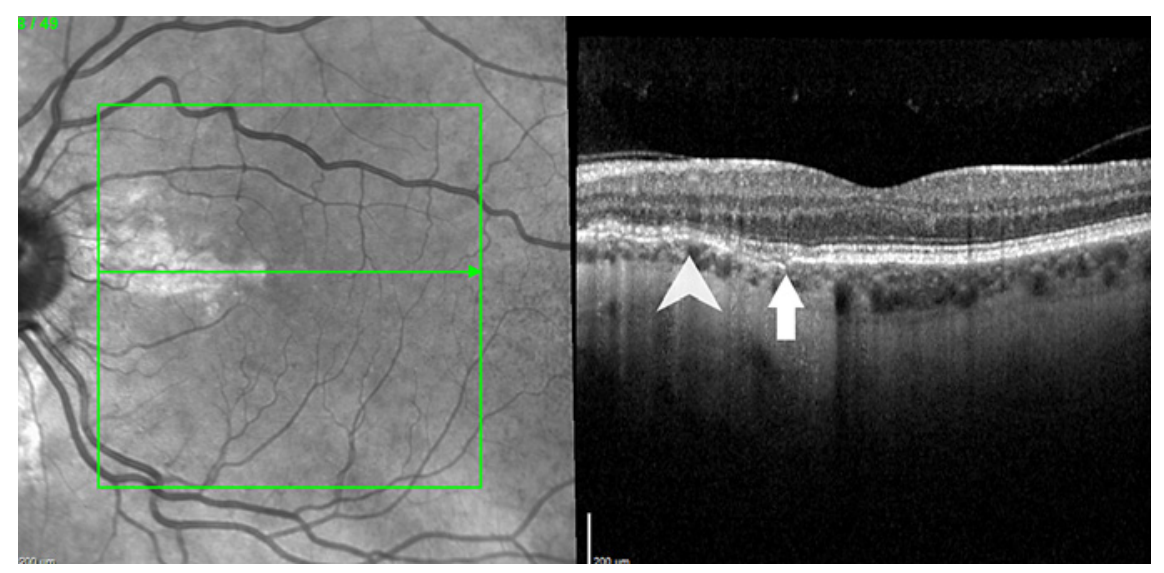

Fig. 3. Spectral domain ocular coherence tomography scan of the subject's left eye localized over an angioid streak shows Bruch's membrane undulation (labelled white arrowhead) and adjacent Bruch's membrane break (labelled white arrow).

\section{Discussion}

In summary, this is a case of AS in a subject with CDA type II. His retinal findings had previously been diagnosed as DFO toxicity, which led to the unnecessary withholding of treatment. This is the second time AS have been reported in CDA type II. AS have been reported more frequently with CDA types I and III.

\section{Pathophysiology of AS}

Histopathology in patients with PXE-associated AS shows calcium deposition in BM. It has also been shown in SD-OCT studies that patients with PXE have increased RPE-BM complex reflectivity, presumably as a result of BM calcification. In Paget's disease bone 

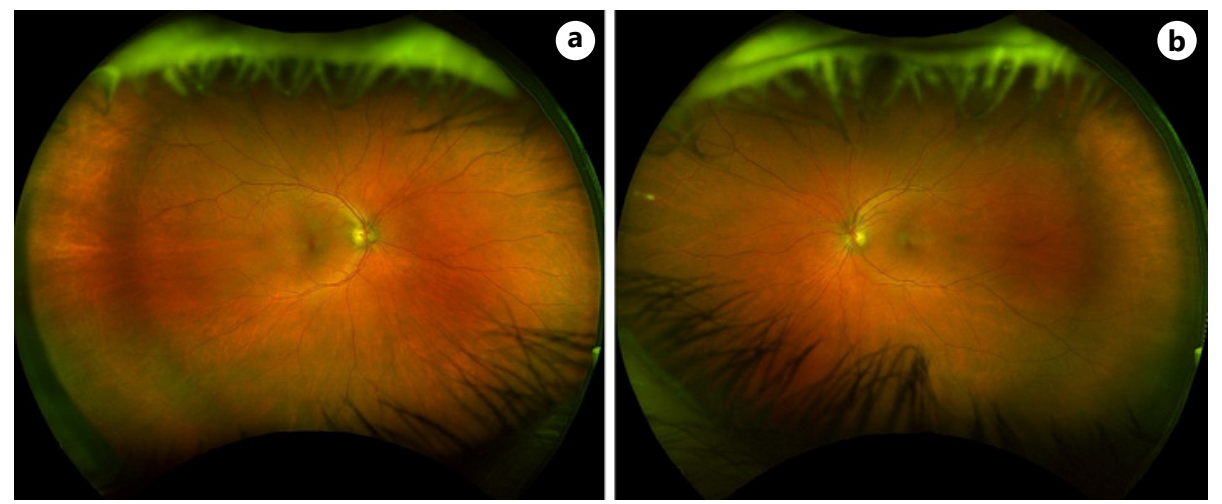

Fig. 4. a Ultra-wide-field confocal scanning laser ophthalmoscopy composite colour image of the subject's brother's right eye, which is unremarkable. b Ultra-wide-field confocal scanning laser ophthalmoscopy composite colour image of the subject's brother's left eye, which is unremarkable.

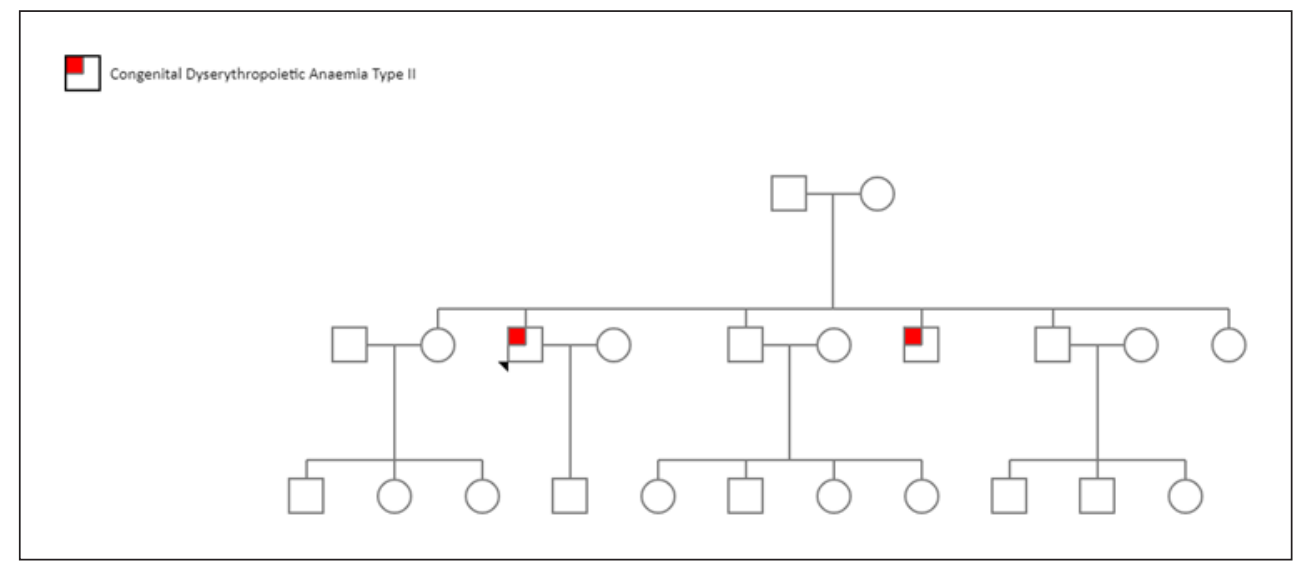

Fig. 5. Family pedigree chart showing our subject (proband, labelled with black arrowhead) and his brother as the only known affected family members.

deformities lead to calcium binding by elastic fibres, suggesting a similar pathophysiology for the association of AS. It has been hypothesized that brittle, calcified BM is more vulnerable to rupture by physiological mechanical forces (e.g., extraocular muscle contraction, minor trauma, intraocular pressure spike) due to its reduced elasticity [1]. BM undulations are a common finding in eyes with AS. In one study that followed up 32 eyes with AS, it was noted that BM breaks most often developed at the undulation vertex and margins. Therefore, undulations represent points of reduced resistance at higher risk of rupture [2].

Initially, the appearance of AS in sickle cell haemoglobinopathies was attributed to high serum iron levels. Increased iron staining of BM in AS was noted in an early report, and AS have been reported in primary haemochromatosis cases. However, AS are not present in other types of anaemia with high serum iron levels. More recent histochemical and electron microscopy studies in eyes of sickle cell anaemia patients confirmed high levels of tissue calcification, which supports the theory that AS in haemoglobinopathy patients are associated with calcium deposition in BM, similar to PXE patients [1].

\section{Karger'}


The question of whether the AS reported in CDA patients are directly related to the CDA mutation cannot be answered with certainty based on the small number of case reports to date. The question of why our subject has AS and his brother does not may represent variable penetrance of AS in CDA. If iron overload plays a role in the development of AS in CDA patients, this may explain why our subject's brother does not exhibit the finding. They rarely occur in patients under the age of 10 years [1], and he underwent a splenectomy at the age of 8, with no treatment required after this. Splenectomy does not prevent iron overload but can reduce it if there is a subsequent reduction in number of required transfusions. Of the 2 CDA II patients with AS which have been reported in the literature thus far; both had splenectomy but not until their third decade, and one remains on DFO treatment [7]. It has been found in beta thalassaemia patients that AS are more likely to be found in patients who have had a splenectomy. This finding is likely to reflect a more severe course of disease in these patients [9].

\section{DFO Toxicity}

Our main differential diagnosis in this case, and indeed the diagnosis our subject was given previously, was DFO toxicity. The mechanism of DFO toxicity has been extensively studied but remains poorly understood. Histologic and ultrastructural studies have suggested that DFO-iron complexes form and induce mitochondrial calcifications of the RPE. These calcifications induce a loss of RPE microvilli and subsequently a thickening of BM, ultimately impairing RPE function. This results in a pigmentary retinopathy, toxic bullseye maculopathy, or pattern dystrophy-like changes [10].

Early RPE changes in DFO toxicity can be easily missed on clinical exam, but may be more easily detected with multimodal imaging. FAF is perhaps the most useful aid to diagnosis. Changes on FAF imaging have been classified into 4 different patterns; minimal change, focal, patchy, and speckled, in descending order of frequency. Changes are bilateral but asymmetrical. No association was found between pattern type and duration of treatment. Patients with minimal change were found to be younger, so it was hypothesized that these minimal changes may progress to one of the other patterns over time [10]. No longitudinal studies exist as yet to confirm this. In one study with an average follow-up of 20 months, eyes with focal changes showed minimal progression over time, independent of whether DFO was continued or discontinued. Patchy or speckled patterns were more likely to develop progressive, enlarging RPE atrophy. Thus the differentiating of these FAF patterns has important prognostic implications [10].

Electrodiagnostic tests have been widely used to confirm the diagnosis of DFO toxicity, with pattern ERG and multifocal ERG most likely to reveal decreasing amplitudes over time. These changes may be reversible with cessation of treatment. Early SD-OCT changes include focal thickening of the RPE, resembling basal laminar drusen, and subretinal hyperreflective deposits. Later changes include RPE and outer retinal atrophy. As mentioned, pattern dystrophy-like changes, most commonly vitelliform, can also occur [10].

Our subject has also been treated with deferasirox, one of a family of newer chelating agents which have largely replaced DFO due to lesser systemic side effects. As a comparatively new drug, there is limited knowledge about its ocular toxicity, consisting mainly of anecdotal case reports of reversible maculopathy [11,12].

Could our subject's long-term treatment with chelating agents contribute to the formation of AS? It has been found in beta thalassaemia patients that AS are more likely to be found in those requiring multiple blood transfusions, and consequently those being treated with chelating agents, although this more likely reflects a more severe disease course [9]. As the pathogenesis is thought to be similar to that of AS, DFO therapy could theoretically contribute to their formation also. However, a detailed review of the manifestations of DFO toxicity did not report a link to AS development [10].

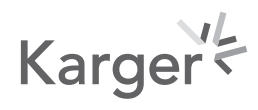


Although unlikely to be a contributing factor in AS, DFO toxicity is an important differential that needs to be out ruled in CDA patients. General ophthalmologists will have an awareness of the risk of DFO toxicity in patients treated with chelating agents. However, due to the rarity of AS reports in CDA patients they may not consider them in the differential when assessing retinal changes. This diagnostic confusion can lead to the withholding of vital chelating treatment, as it did in our subject. If DFO treatment is then withheld, this can lead to an inappropriate discharge from ophthalmological services, when in actuality patients with AS need ongoing monitoring for the development of CNVM.

\section{Conclusion}

To the best of our knowledge, this is only the second case report of AS associated with CDA type II. This is in spite of CDA type II being the most common of the 3 classical CDA types. The pathophysiology of AS in this condition remains poorly understood, and is likely influenced by the clinical course and severity. It is suggested that all CDA patients undergo ophthalmologic examination to screen for both AS, which can have devastating visual consequences, and the complications of chelating treatment. Raising awareness of the occurrence of AS in CDA patients among ophthalmologists may help to avoid diagnostic confusion between these 2 entities.

\section{Statement of Ethics}

This study protocol was reviewed and the need for approval was waived by the Ethics and Medical Research Committee at the Royal Victoria Eye and Ear Hospital. Written informed consent was obtained from both subjects for publication of this case report and accompanying images. Written consent forms and ethical approval waiver are available for viewing.

\section{Conflict of Interest Statement}

There are no potential conflicts.

\section{Funding Sources}

There were no grants or funds received in support of this study.

\section{Author Contributions}

First/corresponding author was responsible for drafting the manuscript. Second author edited and had final approval on the manuscript to be submitted

\section{Data Availability Statement}

All data generated or analysed during this study are included in this article. Further enquiries can be directed to the corresponding author. 


\section{References}

1 Georgalas I, Papaconstantinou D, Koutsandrea C, Kalantzis G, Karagiannis D, Georgopoulos G, et al. Angioid streaks, clinical course, complications, and current therapeutic management. Ther Clin Risk Manag. 2009; 5(1):81-9.

2 Chatziralli I, Saitakis G, Dimitriou E, Chatzirallis A, Stoungioti S, Theodossiadis G, et al. Angioid streaks: a comprehensive review from pathophysiology to treatment. Retina. 2019;39(1):1-11.

3 Hamilton AM, Pope FM, Condon PI, Slavin G, Sowter C, Ford S, et al. Angioid streaks in Jamaican patients with homozygous sickle cell disease. Br J Ophthalmol. 1981;65(5):341-7.

4 Sandström H, Wahlin A, Eriksson M, Holmgren G, Lind L, Sandgren O. Angioid streaks are part of a familial syndrome of dyserythropoietic anaemia (CDA III). Br J Haematol. 1997;98(4):845-9.

5 Roberts E, Madhusudhana KC, Newsom R, Cullis JO. Blindness due to angioid streaks in congenital dyserythropoietic anaemia type I. Br J Haematol. 2006;133(5):456.

6 Tamary H, Offret H, Dgany O, Foliguet B, Wickramasinghe SN, Krasnov T, et al. Congenital dyserythropoietic anaemia, type I, in a Caucasian patient with retinal angioid streaks (homozygous Arg1042Trp mutation in codanin-1). Eur J Haematol. 2008;80(3):271-4.

7 Frimmel S, Kniestedt C. Angioid streaks in types I and II congenital dyserythropoietic anaemia (CDA). Klin Monbl Augenheilkd. 2016;233(4):482-7.

8 Gambale A, Iolascon A, Andolfo I, Russo R. Diagnosis and management of congenital dyserythropoietic anemias. Expert Rev Hematol. 2016;9(3):283-96.

9 Barteselli G, Dell'arti L, Finger RP, Charbel Issa P, Marcon A, Vezzola D, et al. The spectrum of ocular alterations in patients with $\beta$-thalassemia syndromes suggests a pathology similar to pseudoxanthoma elasticum. Ophthalmology. 2014;121(3):709-18.

10 Di Nicola M, Barteselli G, Dell'Arti L, Ratiglia R, Viola F. Functional and structural abnormalities in deferoxamine retinopathy: a review of the literature. Biomed Res Int. 2015;2015:249617.

11 Walia HS, Yan J. Reversible retinopathy associated with oral deferasirox therapy. BMJ Case Rep. 2013;2013: bcr2013009205.

12 Pan Y, Keane PA, Sadun AA, Fawzi AA. Optical coherence tomography findings in deferasirox-related maculopathy. Retin Cases Brief Rep. 2010;4:229-32. 